\title{
NON-SPECIFIC SUPPURATIVE PNEUMONIA
}

\author{
BY
}

\author{
A. LOGAN AND H. NICHOLSON \\ From the Department of Surgery, University of Edinburgh, and \\ University College Hospital, London
}

In 1938 Scadding described a condition which he called chronic suppurative pneumonia, characterized by "irregular consolidation, with incidental breaking down of a small area into an abscess cavity, and the tendency to spread in the form of fresh areas of consolidation which may or may not break down into abscesses." He pointed out that those who have studied large groups of cases of pulmonary abscess find that many abscesses arise without evidence either of aspiration or of embolism, and suggested that some of these should be regarded as chronic suppurative pneumonia.

Touroff and Neuhof (1938, 1941) described, under the title of "non-putrid pulmonary abscess," a condition similar to that described by Scadding. They regarded it as being "initiated by aerobic organisms," whereas anaerobic organisms were responsible for the production of acute putrid lung abscess.

This separation of abscesses into putrid and non-putrid varieties was accepted by Barrett (1944), but Sellors and others (1946) did not consider it correct. In a series of 27 cases, which they described under the name of spreading suppurative pneumonitis, at some time during the illness the sputum was foetid in all but six. The cases in this series showed spreading pulmonary consolidation with cavitation, and a cough with purulent sputum; in 13 of the group the onset was acute, and in all instances the disease ran a protracted course with exacerbations.

The following description is based on a series of cases which showed many of the features described by the authors referred to above. The cases were observed between 1941 and 1945 in a Royal Army Medical Corps thoracic surgical unit in the Middle East.

\section{Clinical and Radiological Features}

The 25 cases which were observed occurred in patients of 11 nationalities; nine were British, four Italian, three West African, two Yugoslav, and one each German, Polish, Greek, Cypriot, Indian, Arab, and Cape Coloured. All were males and their ages were between 18 and 43 years. Because they were all in some form of military service, they came under medical care within a few weeks of the onset of illness. The periods for which they were under observation ranged between three and 21 months, the average being nearly 10 months.

There was no history of previous notable pulmonary illness and there was no reason to assume that there had been inadequate nutrition in any case. In one patient the onset of the disease followed a bilateral Caldwell-Luc operation. In another it followed the repair of an extensive bullet wound of the palate, and in a third it followed anaesthesia of the left side of the tongue, cheek, and palate due to a cranial nerve injury. In a fourth case coma due to a fractured skull preceded the onset of symptoms. Two of the others had gingival suppuration, but in the remaining 19 cases there was no abnormality of the upper respiratory tract to which the onset could be related.

In 15 cases the onset was acute, with pain in the chest, cough, and fever as the main features. In the remaining 10 cases the onset was more gradual, consisting usually of cough and increasing fever. There was copious sputum from the beginning in all except one case, in which, for the first four months, only traces of mucopus were expectorated. Apart from this, frankly purulent sputum, described in 13 instances as foetid, was coughed up from the outset.

The initial febrile period did not usually persist for more than a few weeks, but all cases were characterized by repeated bouts of fever, lasting a week or two, in which the temperature rose to the vicinity of $101^{\circ} \mathrm{F}$. or, rarely, to higher levels. Between these attacks most patients were able to get about and were often in fairly good general condition. Cough was present in all cases and was productive of purulent sputum in amounts which ranged between 50 and $1,100 \mathrm{ml}$. in 24 hours. In only four of the cases was the sputum non-foetid throughout the period of observation.

Haemoptysis was fairly frequently a feature of the condition. It was noted by three of the patients at the onset when it consisted of streaking of the sputum. During the course of the illness, one patient had a haemoptysis of approximately 
$600 \mathrm{ml}$., two others had haemoptysis of $120 \mathrm{ml}$., and a fourth had repeated staining of his sputum.

Twenty-three of the patients had finger clubbing, and one of these had hypertrophic pulmonary osteo-arthropathy. Physical signs suggesting pulmonary consolidation were present at some time in most cases, but the signs varied during the course of the disease and were seldom as gross as might have been expected from the radiological appearances.

The total white cell count was not often much above normal. The highest recorded was 19,800 per c.mm., and only seven patients had at any time a leucocytosis of more than 12,000. The differential count showed approximately normal percentages in all cases. On examination of the sputum a mixed growth of organisms was obtained in each case. The organisms were distributed fairly evenly among the following types: Streptococcus viridans, Streptococcus haemolyticus, Micrococcus catarrhalis, pneumococcus, and Staphylococcus aureus. On two occasions coliform organisms were also detected. In each case at least 12 sputum specimens were examined for the tubercle bacillus and found to be negative, and at least one specimen was cultured on Loewenstein's medium with negative result.

In five cases bronchoscopy was not carried out, either because the patient was regarded as being too ill or because he was not capable of adequate co-operation. In the remaining cases redness and oedema of the mucous membrane of the bronchi associated with the affected areas were observed, and there was usually pus welling out of these bronchi.

Radiography showed that, at the onset, there was consolidation of segmental distribution in 12 cases. In seven cases a whole lobe was involved, and in one there was consolidation of most of one lung. Consolidation scattered throughout a lobe was seen in two cases. Three patients did not have radiographs made sufficiently early in the course of the disease to demonstrate the original distribution of their lesions.

Of the 22 cases in which radiographic evidence of the original situation of the disease was available, 15 began in the right lung and seven in the left. The whole of the right upper lobe was involved at the onset in two cases, its anterolateral segment in two, its postero-lateral segment in one, and the axillary parts of both of these in three. The middle lobe and the adjacent part of the upper were the site of origin in two cases, and the middle lobe and the adjacent part of the lower in another two. The whole of the right lower lobe was involved at the beginning of one case, its apical segment in one, and its posterior basic segment in another. Of the cases which began on the left side, one involved the whole lung at the outset, three, most of the upper lobe, and one, most of the lower; the postero-lateral segment of the upper lobe and the apical segment of the lower were the sites of origin of the two remaining cases.

In five instances a cavity appeared within three weeks of the onset of symptoms. In a further nine there was cavitation within three months of the onset, and, in five more, cavities were not seen to appear until later. There were six cases in which cavitation was not seen at any stage.

The behaviour of the lesions, as followed in radiographs taken during the course of the disease, was varied. In general the disease spread by direct extension from the original lesion into the surrounding lung. This spread was apparently not hindered by interlobar septa. The radiological appearance frequently remained stationary at this stage for two or three months, although clinically the patient often improved. A temporary clearing of the consolidation occurred not uncommonly, but was followed almost invariably by recurrence of the consolidation and spread to other parts of the same lobe or to other lobes of the same lung. In only one case was there a spread to the opposite lung. Spread to other parts of the same lobe or to other lobes was often clearly by direct extension, but spread to parts not in direct continuity occurred in seven cases.

\section{Pathological Features}

An opportunity for dissection of the diseased segment of lung was presented in 12 cases either after ${ }^{\circ}$ its surgical removal or at post-mortem examination. Similar naked-eye and microscopic characters were observed in all. The affected lung was for the most part airless, grey, and of rubbery consistency, and contained one or more cavities $0.5 \mathrm{~cm}$. to $3.0 \mathrm{~cm}$. in diameter. The related pleura was thick and shaggy from adhesions and interlobar fissures were obliterated. In a few, dilated bronchi ringed with fibrous tissue were recognizable. Microscopically, the cavities were lined with granulation tissue showing more or less advanced fibrosis and infiltrated with plasma cells and lymphocytes. In some there was a partial lining of columnar or squamous epithelium. The surrounding alveoli were filled with pneumonic exudate which in some was undergoing organization. Groups of alveoli were lined with cubical cells and there were patches of emphysema. There were occasional abscess-like masses of polymorphs. 
The bronchi showed at some points complete necrosis of their walls, at others inflammatory infiltration and at others fibrosis. The microscopic changes were in no way distinguishable from those occurring in relation to subacute or chronic lung abscess.

\section{TREATMENT}

The 25 patients came under our care at periods which ranged between three weeks and nine months after the onset of the disease. In 14 instances this period was more than three months. On admission, 13 patients had cavitation in the diseased area ; in four there were several cavities present.

All patients were treated by postural drainage under the care of a physiotherapist. They all had repeated courses of one or other of the sulphonamides. Eight patients were given penicillin, the others being under our care before this antibiotic was available. Following these measures one patient became free from symptoms and the radiograph of his chest showed no more than a small amount of residual fibrosis. Another became well, with a normal radiograph, but continued to have $30 \mathrm{ml}$. of purulent sputum each day. There were also two patients who after prolonged illness became well and were left with only traces of sputum and radiological changes indicating gross fibrosis. Bronchograms were carried out and showed distorted and dilated bronchi on the diseased side. The rest improved clinically but relapsed afterwards, and it was not observed, apart possibly from the four cases mentioned, that chemotherapy had any effect on the progress of the disease.

External drainage of cavities was carried out in six cases. In no case did this procedure benefit the patient, and the suppurative process continued to spread in the lung. In one case a left upper thoracoplasty was carried out. This case began with consolidation of the whole of the left lung and an abscess cavity in the axillary part of the upper lobe. This cavity was drained. The lower lobe opacity cleared, but clinically the patient was not improved and he continued to cough up $240 \mathrm{ml}$. of purulent sputum each day. The inflammatory process spread again to his lower lobe and a cavity appeared in its apical segment. Eight months after the drainage operation he was still very ill. Thoracoplasty was then carried out, and following the operation he was better clinically and his sputum was reduced to $150 \mathrm{ml}$. in the day. The cavities were still present and it was not thought that his outlook had been improved by the operative procedures.
Lobectomy was carried out in nine cases and pneumonectomy in one. There were four deaths following operation, one being due to air embolism, one to operative shock, one to anoxia produced by blockage of the intratracheal tube with mucopus, and one to obstructive collapse of the contralateral lung produced by purulent sputum. Five of the remaining patients became completely well and free from sputum following the operation; the sixth was well but had a trace of sputum. In none of this group of six cases was there evidence of residual pulmonary suppuration.

Besides the four deaths which followed operation there were two others. One of these was due to cerebral abscess and one to overwhelming infection.

Thus, of the series of 25 patients, six died. Seven were regarded as having a satisfactory result, this result being achieved in six by pulmonary resection. One was greatly improved; radiographs of his chest were normal and his sputum was reduced to $30 \mathrm{ml}$. each day. Two were left with fibrotic, bronchiectatic lungs, although clinically well. The remaining nine patients, at the end of the period over which they were observed, continued to be ill with a large quantity of sputum and extending inflammatory changes in their lungs.

\section{Illustrative Cases}

Case 1.-An English officer in the Pioneer Corps, aged 33, was admitted on Feb. 8, 1945. He had had bronchitis in November, 1944, but had apparently recovered completely. $\mathrm{He}$ had been admitted to another military hospital on Jan. 2, 1945, a day or two after he had got a soaking in a rainstorm. He had a high temperature and a cough. Clinically he had widespread evidence of bronchitis over the right side of his chest. His sputum a few days later was noted to be purulent, about $90 \mathrm{ml}$. in amount. Radiographs taken at this time showed gradual development of an opacity occupying the whole of the right upper lobe.

On admission he was in good general condition with $90 \mathrm{ml}$. of purulent, but not foetid, sputum each day, and was irregularly febrile to $100^{\circ} \mathrm{F}$. His fingers were clubbed. Over his right upper chest he had physical signs suggesting consolidation. There were no abnormal physical signs elsewhere. Radiographs taken on Feb. 10, 1945 (Figs. 1 and 2) showed consolidation of the right upper lobe, in which there were translucent areas suggesting cavities. A white cell count showed 11,000 leucocytes per c.mm., of which $61 \%$ were polymorphs. Examination and culture of the sputum showed the presence of Micrococcus catarrhalis, diphtheroids, and Staphylococcus aureus; a few colonies of a haemolytic streptococcus were also grown. Bronchoscopy on Feb. 19 showed a normal 


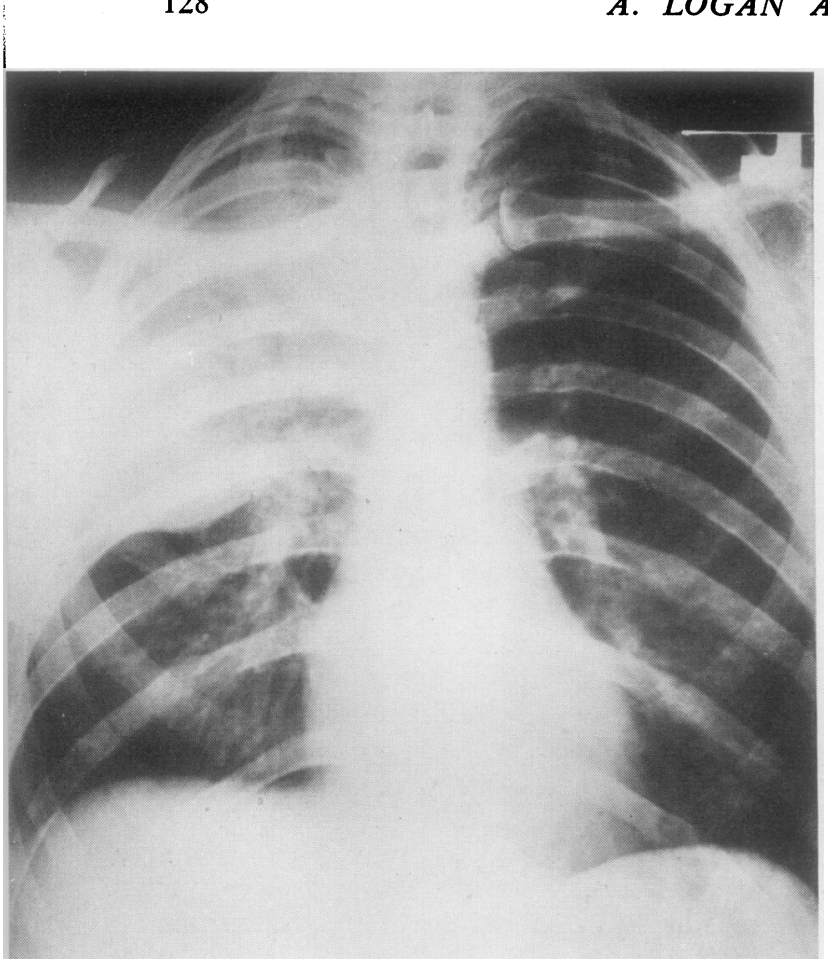

FIG. 1.

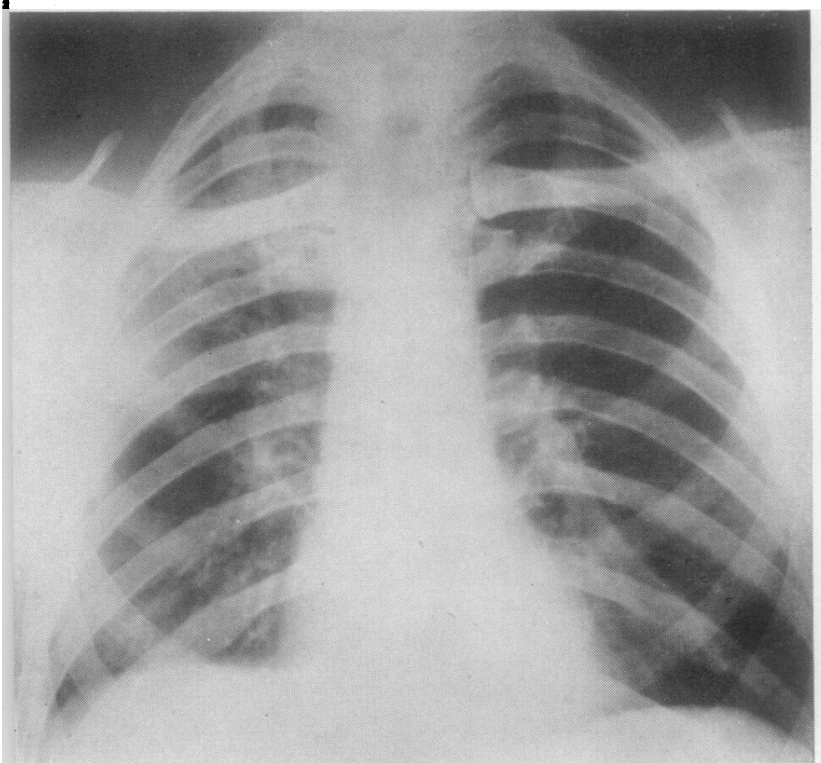

FIG. 3.

FIG. 1.-Case 1: Radiograph taken Feb. 10, 1945.

Fig. 2.-Case 1: Radiograph taken Sept. 4, 1943.

FIG. 3.-Case 1: Radiograph taken March 5, 1945.

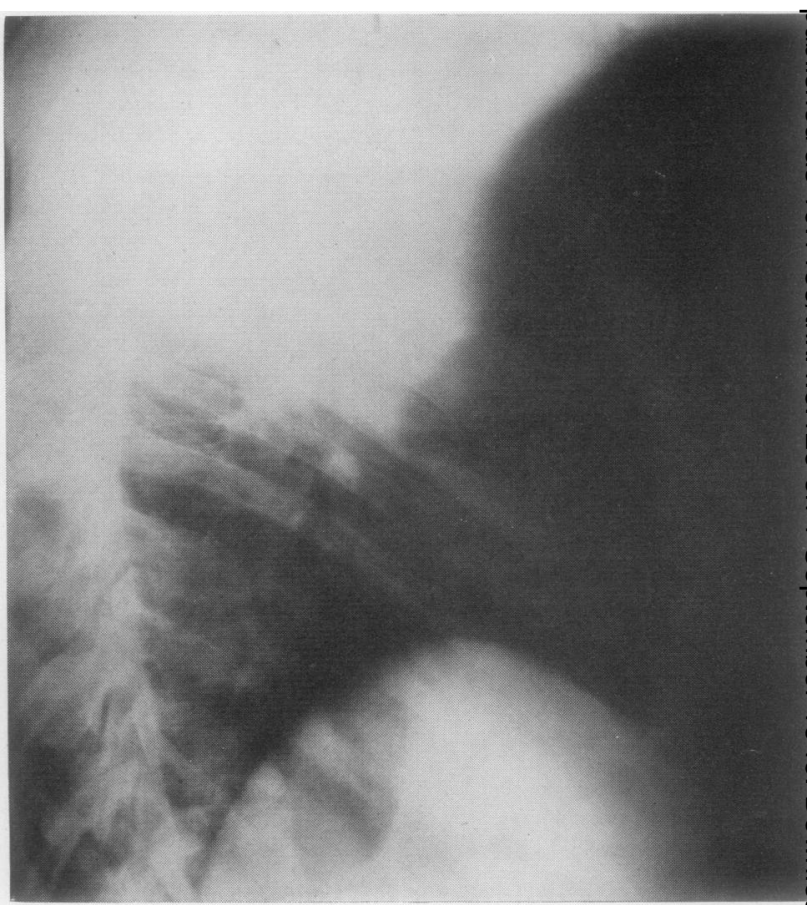

FIG. 2.

bronchial tree apart from some oedema of the right stem bronchus. There was pus lying in the right upper lobe bronchus, and a specimen was aspirated for bacteriological examination. From this specimen non-haemolytic streptococci were grown on culture, but culture on Loewenstein's medium did not grow tubercle bacilli after a month's incubation. Examination of 12 sputum specimens for the tubercle bacillus was also negative.

For seven days after Feb. 20 he was given a course of $43 \mathrm{~g}$. of sulphamezathine and penicillin (approxiniately $1,000,000$ units). A radiograph on March 5 (Fig. 3) showed considerable clearing of the right upper lobe opacity. Beginning on March 7 the course of sulphamezathine and penicillin was repeated. On March 14 the patient complained of pain over his right lower chest and friction was heard there. A radiograph on March 24 showed a small effusion in the right costophrenic angle, but further clearing of the opacity in the upper lobe. The patient was at this time very well, afebrile, and with his cough and sputum decreasing in amount. This improvement continued, and on May 26 he was free from cough and sputum. His radiographs (Figs. 4 and 5) showed flattening of the right diaphragm with obliteration of the costophrenic angle. There appeared to be a little residual fibrosis throughout the right upper lobe.

Case 2.-A Libyan Arab, aged 26, was admitted on June 26,1942 . He had been in another hospital from Feb. 18, 1942, until transferred to our care. His history was that in January, 1942, he had become ill 


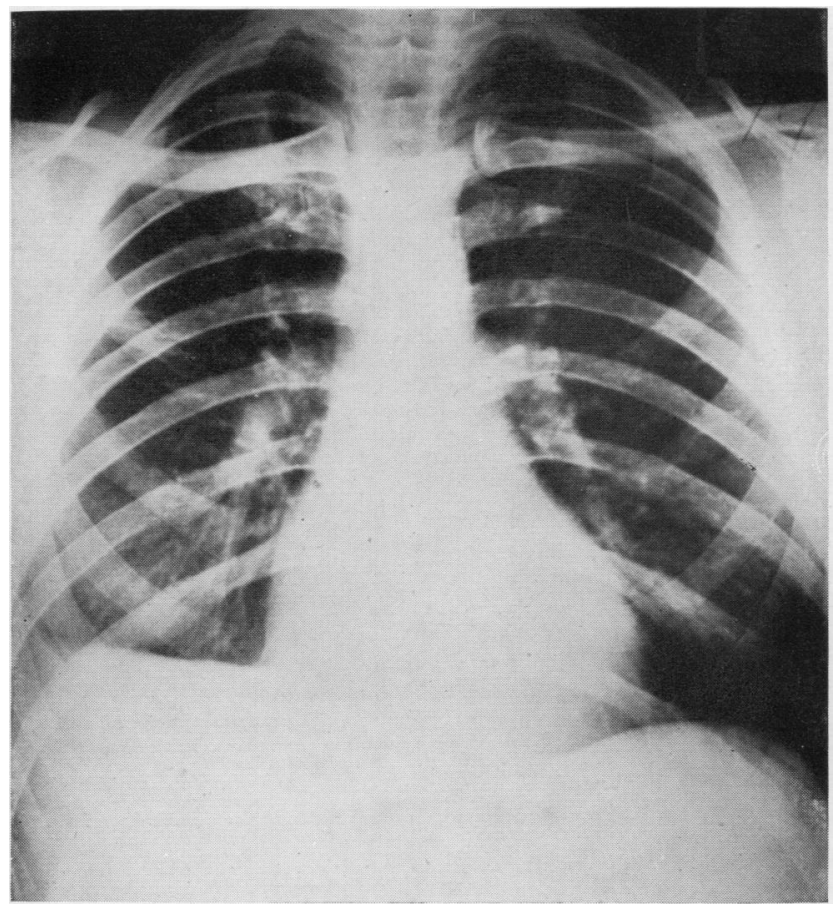

Fig. 4.

with fever, cough, and sputum. The onset of this illness was not acute, and his sputum gradually increased in quantity until in March, 1942, it was recorded that he was bringing up $420 \mathrm{ml}$. to $600 \mathrm{ml}$. of purulent sputum each day. Radiographic examination at this time showed a right upper lobe cavity surrounded by a large area of consolidation in which there were probably other smaller cavities.

On admission his general condition was fairly good. His temperature rose in the evenings to $101^{\circ} \mathrm{F}$. and he had a severe cough with $300 \mathrm{ml}$. of purulent foetid sputum each day. His fingers were clubbed. Abnormal physical signs were confined to the right upper chest, where the percussion note was impaired and the breath sounds were harsh. Radiographic examination revealed that the cavity was smaller, but much of the upper lobe showed consolidation and fibrosis. The transverse fissure was displaced upwards (Fig. 6). A white cell count showed 11,700 leucocytes per c.mm., of which $74 \%$ were polymorphs. Sputum examination showed the presence of a mixed flora with pneumococci predominating ; numerous examinations for the tubercle bacillus made during his stay ir hospital were negative. Bronchoscopy was carried out on June 29,1942 ; the right stem bronchus was found to be oedematous with pus exuding from the eparterial bronchus.

During July, 1942, the patient improved clinically and his sputum diminished in quantity. An exacerbation occurred at the end of July and sulphapyridine, 1 g. every four hours, was begun. This was kept up

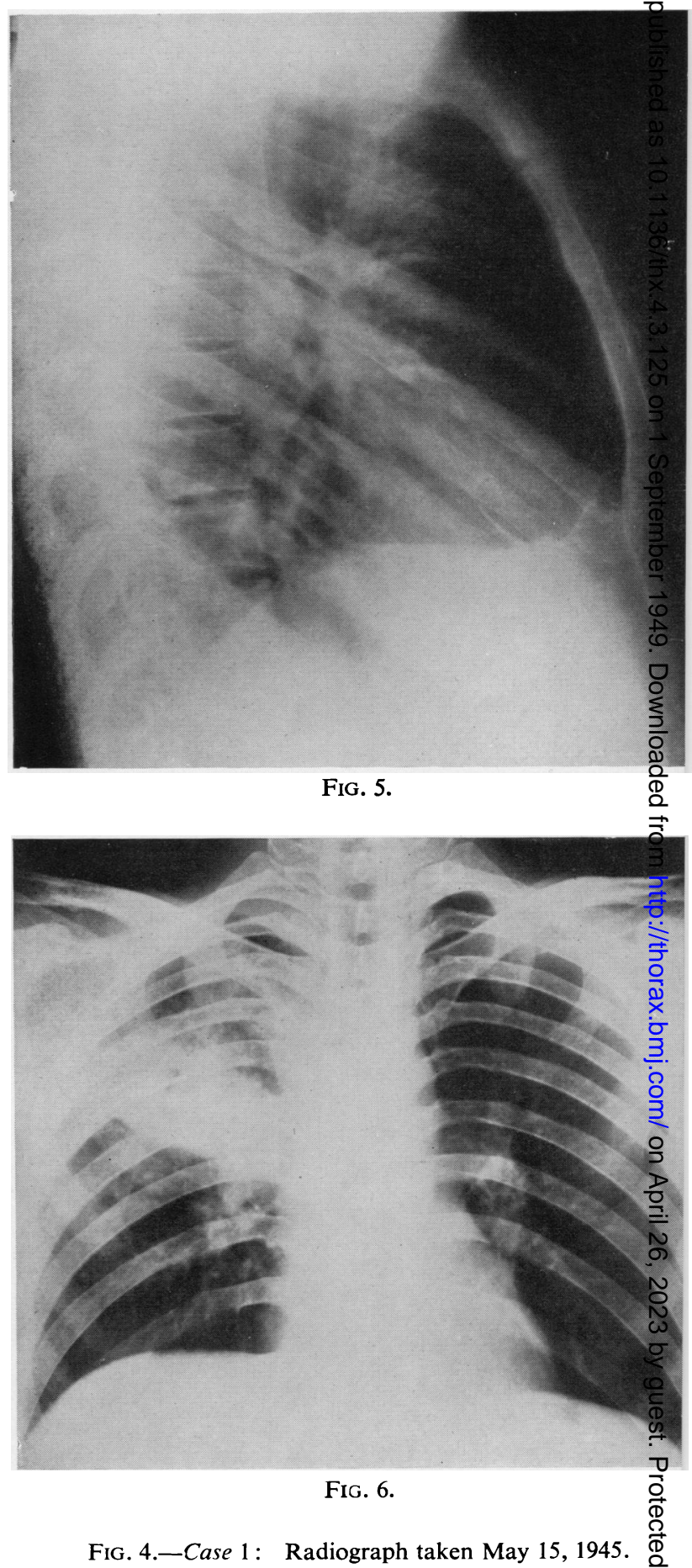

FIG. 5.-Case 1: Radiograph (right lateral view) taken May $15_{1}$ FIc. 6.-Case 2: Radiograph taken July 22, 1942. 
FIG. 7.-Case 2: Radiograph taken Dec. 9, 1942.

Fig. 8.-Case 2: Radiograph taken May 8, 1943.

FIG. 9.-Case 2: Bronchogram taken June 1, 1943.

Fig. 10-Case 2: Bronchogram taken Jnne 1. 1943.

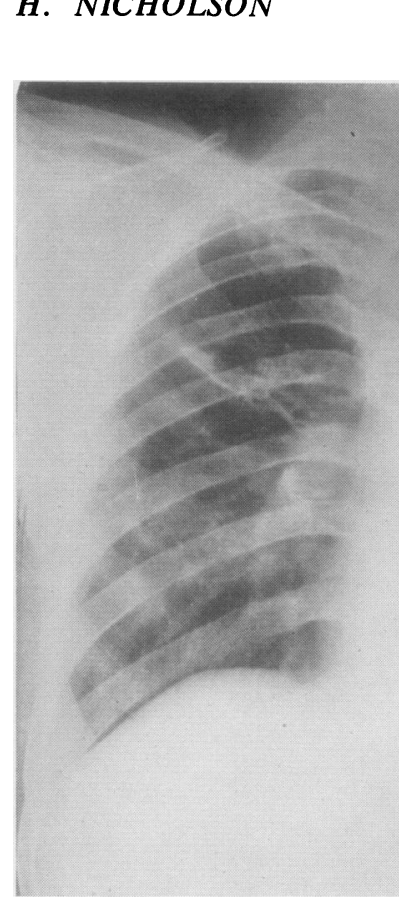

FIG. 7.

FiG. \&.
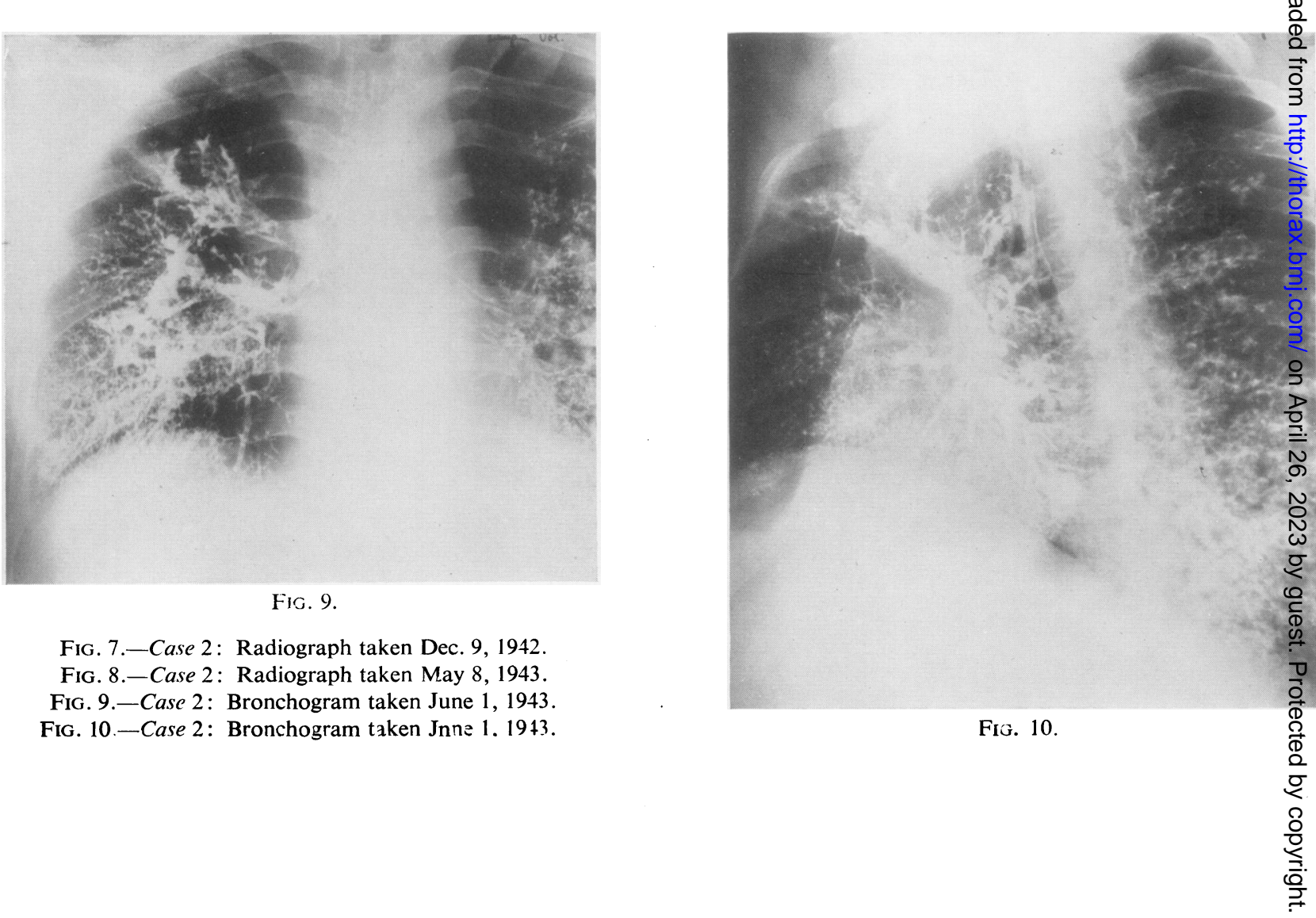
for 18 days, frequent white cell counts being done during this period. He did not suffer from nausea or other ill effects, and at the end of this prolonged course was greatly improved and had only $90 \mathrm{ml}$. of sputum each day. The clinical improvement was maintained, but radiographs in December, 1942, showed that although the upper lobe changes were mainly those of fibrosis there was a large pneumonic area in the right lower lobe (Fig. 7). During January, 1943, the patient became ill again, febrile to $102^{\circ} \mathrm{F}$. and with greatly increased sputum. The area of consolidation in the right lower lobe increased in size, and early in February it was clear that he had a right basal empyema. This was drained and the empyema cavity was rapidly obliterated by complete reexpansion of the lung. The lower lobe at this time had in it two cavities with fluid levels, and much surrounding consolidation.

Following this the patient steadily improved and radiographic examination of his chest showed increasing pulmonary fibrosis. The appearance on May 26 is shown in Fig. 8. He was then free from cough and sputum, and was fat and well. Bronchograms carried out on Jan. 1, 1943 (Figs 9 and 10) showed a distorted right bronchial tree with irregular cavities in communication with the bronchi of all lobes.

Case 3.-A Yugoslav soldier, aged 30, was admitted on Sept. 4, 1943. On May 25, 1943, he had had, in another military hospital, bilateral Caldwell-Luc operations. Ten days later he became febrile and began to expectorate foul-smelling sputum. Radiographic examination on June 21,1943 , showed an opacity in the apical segment of the right lower lobe without obvious cavitation. During July and August, 1943 , his temperature continued to vary up to $99^{\circ} \mathrm{F}$. and he had between $150 \mathrm{ml}$. and $270 \mathrm{ml}$. of foulsmelling purulent sputum each day. On one occasion his sputum was streaked with blood.

On admission his general condition was fairly good ; his evening temperature was in the region of $100^{\circ} \mathrm{F}$. and he had much cough with $1,100 \mathrm{ml}$.' of purulent foetid sputum each day. His fingers were clubbed. There were no abnormal physical signs to be detected in his chest or elsewhere. Radiographs (Figs. 11 and 12) showed a large opacity mainly in the apical segment of the right lower lobe with a cavity at its upper end. A white cell count showed 13,800 leucocytes per c.mm., of which $83 \%$ were polymorphs. Numerous examinations of sputum specimens during the patient's stay in hospital were negative for tubercle bacilli. Bronchoscopy was carried out on Sept. 11, and no abnormality was seen apart from a steady flow of pus from the inflamed orifice of the bronchus to the apical segment of the right lower lobe.

In two stages, on Sept. 13 and Sept. 23, 1943, a free pleura being found at the first operation, the abscess cavity was drained externally. The patient improved after operation and his sputum had decreased to $450 \mathrm{ml}$. each day by the end of September. This improvement was not maintained; by the middle of December he was again febrile to $100^{\circ} \mathrm{F}$., had up to $1,100 \mathrm{ml}$. of sputum a day, and radiographs (Figs. 13 and 14) showed considerable clearing of the opacity in the apical segment but a spread of the inflammatory process mainly to the posterior basic segment of the right lower lobe. His drainage track remained as a sinus communicating with a bronchial fistula. Bronchoscopy was repeated on Dec. 13, 1943, and showed pus exuding from all the branches of the right lower bronchus. After transfusion with 1,200 ml. of fresh blood, a right lower lobectomy was carried out on Jan. 25, 1944. This was followed by a small basal empyema which healed after drainage. The patient was greatly improved immediately after the lobectomy, but he continued for three months afterwards to have a trace of purulent sputum. When last seen on Sept. 27, 1944, he was well, his fingers were not clubbed, and he had no cough. He did, however, produce a little mucoid sputum from his throat each morning. Radiographs and bronchograms showed no abnormality of the remaining right upper lobe.

\section{Discussion}

Distinction from the Specific Pneumonias.-It is generally agreed that the specific pneumonias which are associated with cavity formation present nosological features which distinguish them from the sort of pulmonary suppuration which has just been described. Cavity formation during the course of pneumococcal pneumonia has been reported by Kessel (1930) as a benign process leading to rapid and complete healing.

Chronic pneumonia due to Friedländer's bacillus has been described by Collins and Kornblum (1929) as of confluent lobular distribution, migration to other lobes and multiple abscess formation in the affected lobes being characteristics. In their cases a profuse growth of Friedländer's bacillus was obtained from the sputum. In those of Belk (1926) Friedländer's bacillus was obtained from the sputum, or from the lungs or blood at necropsy.

Staphylococcal pneumonia was described by Heffron (1939) and by Brock (1946). It occurs most frequently following other respiratory infections or during the course of chronic debilitating disease. The onset is usually insidious with a high remittent fever such as is characteristic of staphylococcal infection elsewhere in the body. Multiple cavities develop in the pneumonic areas and Staphylococcus aureus is the predominant organism in the thick purulent sputum. The pulmonary abscesses which occur in the course of staphylococcal septicaemia are also distinguished by the demonstration of staphylococci in almost pure culture in the sputum, or in pure culture in the blood, as well as by their thin-walled, balloon-like appearance in radiographs.

Pulmonary tuberculosis, also a specific pneumonia associated with cavity formation, may be 
FIG. 11.-Case 3: Radiograph taken Sept. 4, 1943.

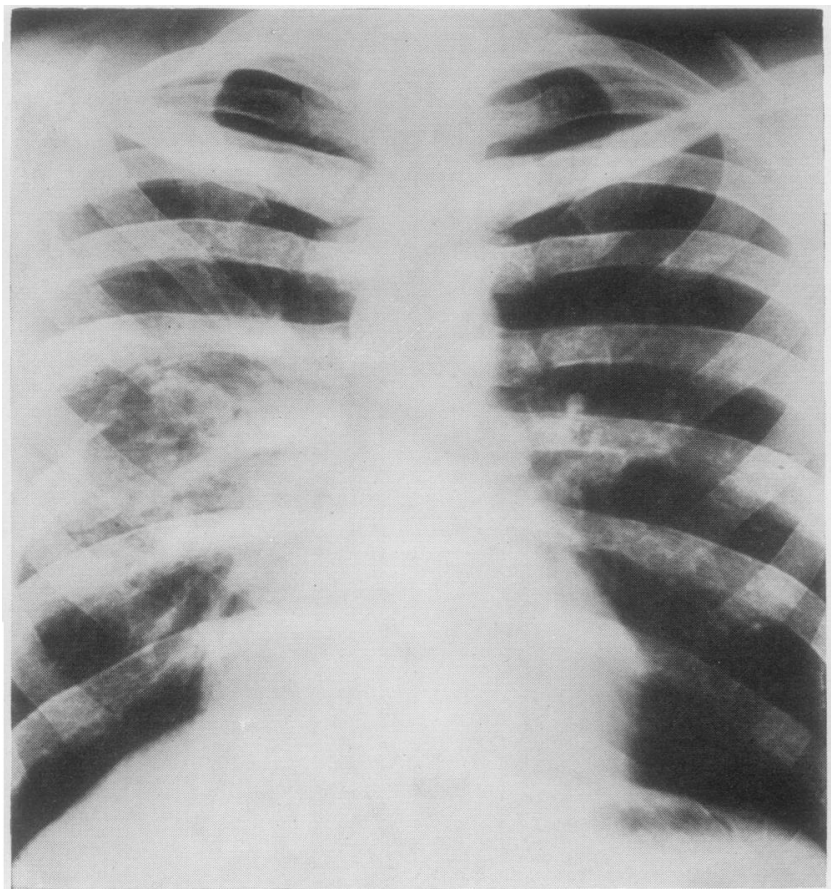

FIG. 13.-Case 3: Radiograph taken Dec. 10, 1943.

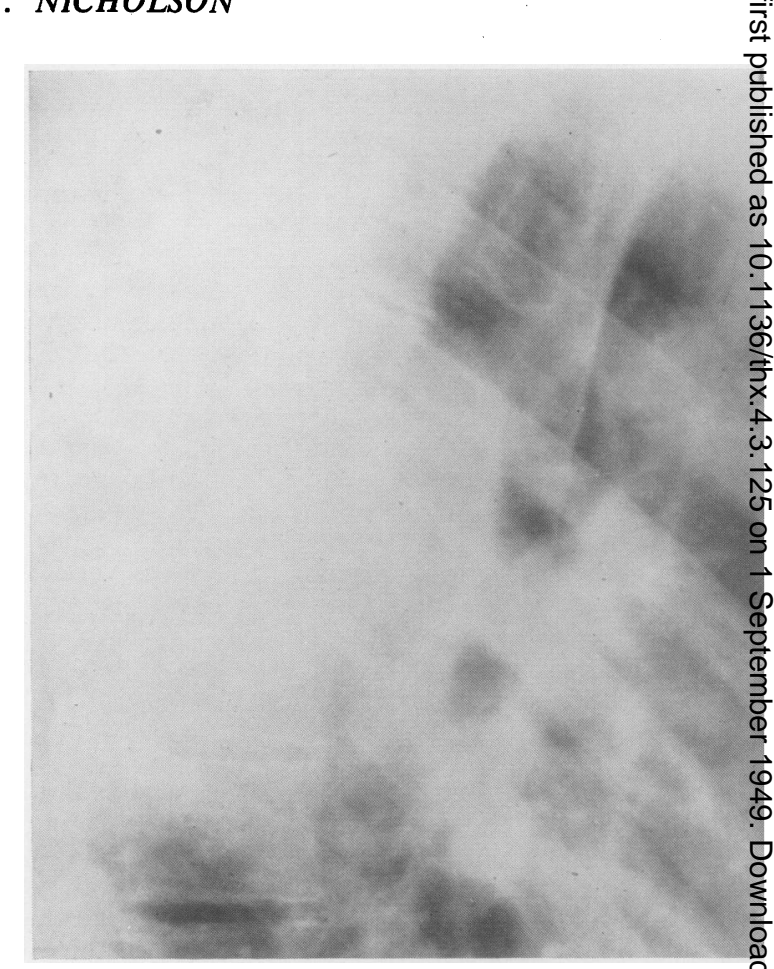

FIG. 12.-Case 3: Radiograph (right lateral view) tak@̣n Sept. 11, 1943.

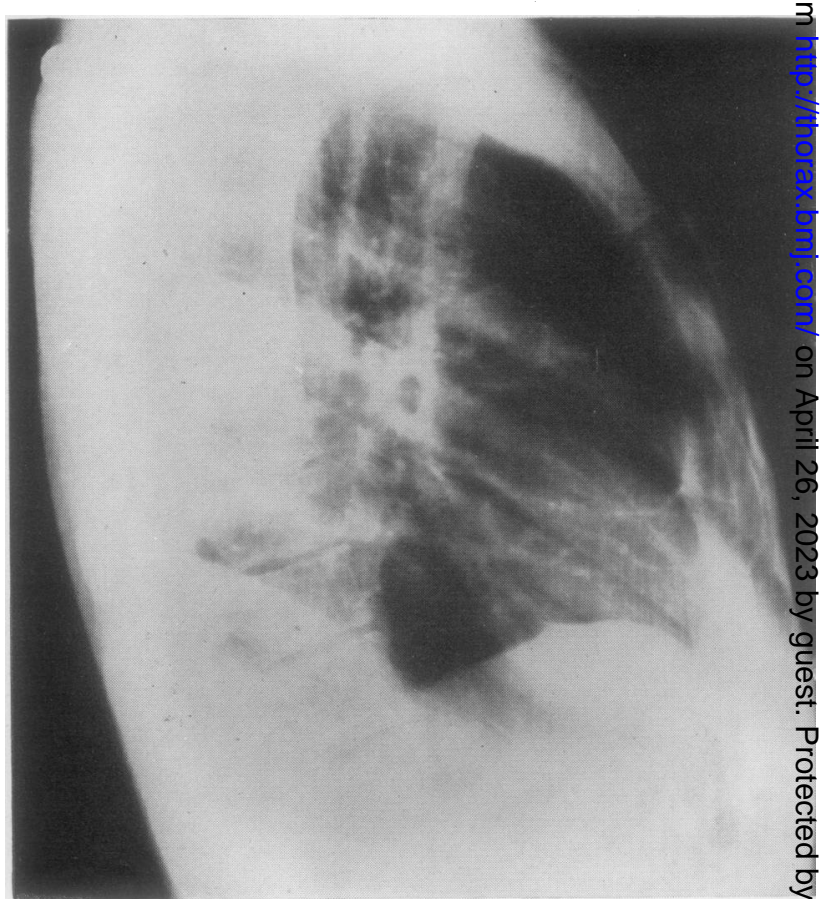

Fig. 14.-Case 3: Radiograph (right lateral view) taken Dec. 13, $19 \mathrm{~g} 3$. 
confused clinically because of its course and its radiological similarities. The purulent sputum in which, on repeated examination, tubercle bacilli cannot be demonstrated, adequately distinguishes suppurative pneumonia from tuberculosis.

Relation to Benign Aspiration Pneumonias and Acute Putrid Lung Abscess.-In the course of catarrhal upper respiratory infections localized aspiration pneumonias are common, and most follow a benign course to complete resolution (Ramsay and Scadding, 1939). These authors suggested, however, that occasionally these usually benign consolidations may progress as chronic suppurative pneumonias. The nature of the bacterial flora of the aspirated mucus may determine whether the affected area of lung should undergo a transitory inflammatory reaction or one with a more prolonged course involving suppuration and fibrosis.

The spread of the inflammatory process in chronic suppurative pneumonia is mainly direct, but spread to distant parts of the lung, as in seven of the cases described, does also occur, presumably by aspiration of pus. When the type of pulmonary suppuration which is usually distinguished as acute putrid abscess becomes complicated by further suppurative changes in the lung, its method of spread and its course do not differ from those of a suppurative pneumonia. In the series we have described there were five cases in which cavitation occurred within three weeks of the beginning of symptoms and was, at the onset, the dominant feature radiologically without much surrounding pneumonia. The subsequent behaviour of these lesions did not differ from that of the rest of the group.

The distinction of non-specific pulmonary suppuration into putrid and non-putrid varieties is made by various authors. For example Touroff and Neuhof (1941) regard this distinction as particularly important as far as treatment is concerned, the treatment of the putrid variety being by surgical drainage and that of the non-putrid variety mainly by chemotherapeutic agents. As Sellors and others (1946) point out, this distinction is not valid since all but six of their cases of spreading suppurative pneumonitis had foetid sputum at some time in the course of their illness. In our series foetor of the sputum was observed at some time during the course of the disease in all but four cases. It would seem more reasonable to regard non-specific pulmonary suppuration as a single disease. At one extreme it consists in its early development of a relatively uncomplicated abscess cavity ; at the other extreme the pneumonic process predominates from the beginning and cavitation may not be apparent at any stage.

Treatment.-The distinction between the extrem? types of non-specific pulmonary suppuration has its only importance in the occasional value of surgical drainage of the relatively uncomplicated abscess. When abscesses occur later in the course of the disease and when pneumonic changes are predominant, surgical drainage of cavities does not materially affect the progress of the condition.

Postural drainage and chemotherapy, as far as the series we have described is concerned, produced little in the way of lasting results, although most patients were benefited temporarily by their use. It seemed that the only procedure by which the patient could be rid of his pulmonary suppuration and freed from the likelihood of further occurrence of it, was by resection of the affected lung or lobe. The value of postural drainage and chemotherapy seemed to be mainly to prepare the patient for pulmonary resection by improving his general condition and by temporarily reducing the activity of the suppurative process in his lung.

\section{SUMMARY}

A description of chronic suppurative pneumonia is given, based on a series of 25 cases. This condition can be clearly separated from those pneumonias of specific aetiology which are associated with cavity formation.

The differentiation of non-specific pulmonary suppuration into putrid and non-putrid varieties is not held to accord with observed facts, and it is suggested that all non-specific pulmonary suppuration is one disease.

When the disease began otherwise than as a relatively uncomplicated abscess, surgical drainage led to uniformly bad results. Of other methods used in treatment, postural drainage and chemotherapy produced, as a rule, only temporary improvement. Resection of the affected lung or lobe was the only satisfactory method of treatment.

\section{REFERENCES}

Barrett, N. R. (1944). Lancet, 2, 647.

Belk, W. P. (1926). J. infect. Dis., 38, 115.

Brock, R. C. (1944). Guy's Hosp. Rep., 94, 115.

Collins, L. H., Jnr., and Kornblum, K. (1929). Arch. intern. Med., 43, 351 .

Heffron, R. (1939). Pneumonia with Special Reference to Pneumococcus Lobar Pneumonia. O.U.P. London.

Kessel, L. (1930). Arch. intern. Med., 45, 401.

Neuhof, H., and Touroff, A.S. W. (1938). Snrgery, 4, 728.

Ramsay, H., and Scadding, J. G. (1939). Quart. J. Med., $8,79$.

Scadding, J. G. (1938). Proc. Roy. Soc. Med., 31, 1259. Sellors, T. H., Blair, L. G., Houghton, L. E., Thompson, V. C., and Pryce, D. M. (1946). Thorax, 1, 146.

Touroff, A. S. W., and Neuhof, H. (1941). J. thorac. Surg., 10, 618. 